\title{
Postinfective Glomerulonephritis (PIGN) in Children Attending a Tertiary Care Centre in Nepal
}

\author{
Poudel DR ${ }^{1}$, Basnet $\mathrm{S}^{2}$, Gami FC ${ }^{3}$
}

\begin{abstract}
Introduction: Post infectious glomerulonephritis (PIGN) is one of the common paediatric kidney disease in developing countries. This study was undertaken to describe the common clinical features, biochemical findings and complications in children with PIGN. Materials and Methods: This was a retrospective descriptive study in which 30 patients admitted with a diagnosis of PIGN at Tribhuvan University Teaching Hospital (TUTH) in a six month period (July 2014 to Dec 2014) were included. Hospital medical records were reviewed for the data collection. Results: Out of 30 patients, 24 (80\%) were between 5 to 15 years of age (mean age of $11.5 \pm 3.3$ ) with male to female ratio of $2.3: 1$. Pedal oedema and hypertension were the clinical features seen in 29 (97\%) and 28 (93\%) patients respectively. Hypocomplementemia was found in 29 (97\%) patients. Recent past history of sore throat seen in 10 (33\%) patients and skin lesions in 12 (40\%). The complications noted were congestive cardiac failure in $5(17 \%)$ patients, rapidly proliferative glomerulonephritis in $3(10 \%)$ and encephalopathy in $1(3 \%)$. Conclusion: The most important clinical and biochemical profile observed in nepalese children with PIGN are the hypertension, pedal edema and hypocomplementemia.
\end{abstract}

Key words: Post infective glomerulonephritis (PIGN), ASO, C3

\section{Introduction}

A GN (Acute glomerulonephritis) is recognized by clinical features of haematuria, fluid overload (edema and hypertension), and some evidence of renal insufficiency (elevation of blood urea nitrogen and creatinine) ${ }^{1}$. Post infective glomerulonephritis (PIGN) is the commonest form of AGN in developing countries ${ }^{2}$. Children from 4-14 years of age are more commonly affected by PIGN and is twice more frequent in males than females ${ }^{3}$. Although nephritogenic beta hemolytic streptococci constitute the commonest cause of PIGN, several other bacteria and viruses have also been implicated ${ }^{4}$. In this study therefore, a terminology PIGN has been used instead of Post streptococcal glomerulonephritis (PSGN). In the absence of highly sensitive diagnostic modality in resource poor countries, the definitive infective etiology cannot be established in many patients presenting with AGN. Since there are no definitive diagnostic criteria for PIGN, the treating clinician usually makes a final diagnosis of
'Dr. Daman Raj Poudel, MBBS, MD, Teaching Assistant, ${ }^{2}$ Dr. Shrijana Basnet, MBBS, MD, Assistant Professor, ${ }^{3}$ Dr. Fakir Chandra Gami, MBBS, MD, Professor. All from the Department of Paediatrics, Institute of Medicine, Maharajgunj, Kathmandu, Nepal.

\section{Address for correspondence: \\ Dr. Daman Raj Poudel \\ Boharatar - 16, Kathmandu, Nepal \\ Tel No; +9779851135861 \\ E-mail: poudeldamanraj@hotmail.com}

\section{How to cite}

Poudel DR, Basnet S, Gami FC. Postinfective Glomerulonephritis (PIGN) in Children Attending a Tertiary Care Centre in Nepal. J Nepal Paediatr Soc 2014;34(3):221-224.

doi: http://dx.doi.org/10.3126/jnps.v34i3.10707

This work is licensed under a Creative Commons Attribution 3.0 License.

\section{(c) (i)}

PIGN after reviewing all clinical features and biochemical parameter and excluding other causes of glomerulonephritis whenever necessary.

This study aims to describe the common clinical features, biochemical findings and complications in children with PIGN admitted to our hospital.

\section{Materials and Methods}

This is a retrospective descriptive study in which 30 patients with a final diagnosis of PIGN at Tribhuvan University Teaching Hospital (TUTH) in six month's period (July 2014 to Dec 2014). Final diagnosis 
was made on the basis of clinical features (such as edema, hematuria, hypertension, oliguria), laboratory analyses (such as deranged renal function, ASO titre, complement level, ANA) with or without recent past history of skin and/or throat infection. Hospital medical records were reviewed for demographic profiles, clinical features, complications, laboratory data and treatment. The data was analysed in descriptive method and results obtained are shown in the form of frequencies along with the percentage values.

\section{Results}

Out of a total of 30 patients, $24(80 \%)$ were between 5 to 15 years of age (mean age of $11.5 \pm 3.3$ ) with male to female ratio of 2.3:1. Twelve (40\%) patients were referred from other hospitals. There were maximum number of patients in the month of November (9) and December (12). Duration of stay in hospital ranged from 2 to 15 days (mean duration of $7.86 \pm 3.84)$.

The common clinical features were swelling of face and/or leg (100\%), hypertension (93.33\%), decreased urine output $(56.67 \%)$ and red coloured urine $(46.67 \%)$. Eleven (36.67\%) patients had other features like fever, abdominal pain, bleeding from nose, cough, difficulty in breathing and seizure. Recent history of sore throat and skin lesion were seen in 33\% patients and $40 \%$ respectively. (Table 1 )

Table 1: Clinical features of patients with PIGN $(n=30)$

\begin{tabular}{|l|c|}
\hline Symptoms/Signs & Number (\%) \\
\hline Swelling of face and/or leg & $30(100 \%)$ \\
\hline Red or cola coloured urine & $14(46.67 \%)$ \\
\hline Decreased urine output & $17(56.67 \%)$ \\
\hline Headache & $11(36.67 \%)$ \\
\hline History of sore throat & $10(33.33 \%)$ \\
\hline History of skin lesion & $12(40 \%)$ \\
\hline Hypertension & $28(93.33 \%)$ \\
\hline Rashes Healed/fresh & $9(30 \%)$ \\
\hline Pedal edema & $29(96.67 \%)$ \\
\hline Others & $11(36.67 \%)$ \\
\hline
\end{tabular}

Among 30 patients, elevated ASO titre was observed in only $60 \%$ of patients. Though there was a history of gross hematuria in $46.67 \%$, microscopic hematuria was observed in $67 \%$ of patients. C3 was sent in all patients and was found to be decreased in $97 \%$. Renal function derangement was seen in only $50 \%$. Serum electrolytes ( $\mathrm{Na}, \mathrm{K}$ ) were found to be normal in all patients. ANA was done in 9 patients (30\%) but was negative in all. (Table 2 )
Table 2: Biochemical profile of patients with PIGN $(n=30)$

\begin{tabular}{|l|c|}
\hline Laboratory parameter & Number (\%) \\
\hline Low Complement (C3) & $29(97 \%)$ \\
\hline Hematuria (RBC> 5/hpf) & $20(67 \%)$ \\
\hline Elevated ASO titre & $18(60 \%)$ \\
\hline Renal derangement & $15(50 \%)$ \\
\hline Electrolytes disturbances & 0 \\
\hline Reactive ANA & 0 \\
\hline
\end{tabular}

Ultrasound of the kidney was done in five patients ( 1 had associated urinary tract infection, 3 had associated pallor, 1 had short stature) and found to be normal. Three patients with clinical suspicion of RPGN (rising creatinine with decreasing urine output) underwent kidney biopsy which showed crescentic glomerulonephritis in all.

Out of 30 patients, nine (30\%) had complications. The complications noted were congestive cardiac failure in $17 \%$, rapidly proliferative glomerulonephritis (RPGN) in $10 \%$ and encephalopathy in $3 \%$ of patients. Four (13.33\%) had associated problems like infected eczema (3.3\%), urinary tract infection (3.3\%) and nephrotic range proteinuria (6.7\%). (Table 3 )

Table 3: Complications of PIGN $(n=30)$

\begin{tabular}{|l|c|}
\hline Complications & Number (\%) \\
\hline Congestive cardiac failure & $5(17 \%)$ \\
\hline RPGN & $3(10 \%)$ \\
\hline Hypertensive encephalopathy & $1(3 \%)$ \\
\hline
\end{tabular}

Diuretic and nifedipine were the antihypertensives used in $97 \%$ and $77 \%$ of patients respectively. Seventy seven percent of the total patients received both the drugs to control hypertension. Hypertension disappeared within 3 to 12 days with mean duration of $6.6 \pm 2.7$ days. Dialysis required in one patient ( $3 \%$ ). Three patients of RPGN received methylprednisolone.

\section{Discussion}

This study was carried out in six month period from July to December among 30 patients admitted at TUTH with final diagnosis of PIGN. To the best of our knowledge, this is the first published study on PIGN among Nepalese population.

The prevalence is higher among school going age of 5 to 15 years. Similar observation were reported by some previous studies ${ }^{3,5,6}$. Regarding sex, we observed more cases of PIGN in male individual. This may be due to the fact that male children are active and 
liable to get infection more than female. In our study, pyoderma associated nephritis with male individual are predominant which are comparable to other studies ${ }^{5,6,7,8}$.

Our study, in accordance with other four studies, showed edema, hematuria, hypertension and low complement level in significant number of patients. .,9,10,11 The depression of $\mathrm{C} 3$ has been found to be the most reliable indicator of PIGN. All of our patients, except one, had significantly depressed C3 level. Similar observation was also seen in a study by Fabiola D Cruz et al $^{12}$. However elevated ASO titre and deranged renal function was seen in only 60 and $50 \%$ respectively. There might be several reasons of not having elevated ASO titre in majority of our patients. Firstly, antecedent infection may not be streptococcal in origin in large number of our patients. Secondly, most of our patients were pyoderma related where we rarely get elevated ASO titre in the blood. Thirdly, anti DNAse $B(A D N-B)$ test, which usually gets elevated in pyoderma associated nephritis, could not be done in our setting because of unavailability of the mentioned test. In a report by Chug K S et al, the utility of ASO titers in patients with acute glomerulonephritis following streptococcal pyoderma is lower, with elevated concentrations in only $57 \%$; however ADN-B is more consistently elevated (90\%) making it the serologic test of choice in this setting ${ }^{13}$.

Some of the previous studies had even made their own criteria while including cases of PIGN. In a study by KhoybarMAetal, poststreptococcal glomerulonephritis was defined as recent onset of haematuria or history of haematuria plus either of the followings or all: oedema, renal insufficiency, hypertension, heart failure, hypertensive encephalopathy, evidence of recent streptococcal infection (positive throat/Swab culture, history of skin infection or pharyngitis or elevated ASO. titre) ${ }^{5}$. In a study by S Rajajee, criteria for APSGN (acute poststreptococcal glomerulonephritis) made were acute onset of edema, oliguria, hematuria, proteinuria, no history of antecedent renal disease and recent history of skin and/or throat infection ${ }^{14}$. However, with the variability of clinical features and lab parameter, we had not made any criteria and final diagnosis of PIGN was made by the treating paediatrician after reviewing all clinical features and laboratory parameters. We observed that blood pressure was controlled within 3 to 12 days with an average of 6.6 days which was not different from other studies ${ }^{5,15}$.

Besides, the typical features, PIGN occasionally manifests with one or more of its complications, while edema and gross hematuria may be absent. In series of studies, the common complications seen were acute pulmonary edema, hypertensive encephalopathy, acute renal failure and nephrotic syndrome ${ }^{5,14,16}$. The most common complications noted in our study were congestive cardiac failure (17\%), RPGN (10\%) and encephalopathy (3\%). In a study by Fabiola D, Cruz et al, hypertensive encephalopathy and congestive cardiac failure were found in $11.3 \%$ and $36.3 \%$ respectively ${ }^{12}$. In our study, only two patients (6.67\%) had nephrotic range proteinuria. However, in a study by Chug KS et al, $14 \%$ of PSGN patient was found to have nephrotic range proteinuria at the onset ${ }^{13}$. Three of our patients $(10 \%)$ had clinical presentation of RPGN, which revealed crescentic glomerulonephritis on renal biopsy. Study by Marques et al showed RPGN in $1.2 \%$ in age group beyond 14 years ${ }^{11}$. Out of 30 cases studied, there was no deaths in the present study. The mortality rate seemed to vary from $<1 \%$ to $13 \%$ in other previous studies ${ }^{7,14}$.

\section{Conclusion}

The most important clinical and biochemical profile observed in Nepalese patients attending this tertiary care centre with a diagnosis of PIGN are hypertension, pedal oedema and hypocomplementemia.

\section{Acknowledgement: Nil \\ Funding: Nil \\ Conflict of interest: None \\ Permission from IRB: Yes}

\section{References}

1. Thomas R.Welch. An Approach to the Child with Acute Glomerulonephritis. Review Article. International journal of pediatrics. 2012; 2012: Article ID 426192, 3 pages

2. Forfer and Arneils. Disorders of Urogenital System. $5^{\text {th }}$ ed. Churchill Livingstone: Text Book of Pediatrics; AGN: p. 960-62.

3. Bernardo Rodriquez, Itube, Sergiomezzano. Acute postinfectious glomerulonephrtis. In: Ellis D.Aver, William E.Harmon, Patrick Nianoet, Norshige Yoshikawa. Pediatric Nephrology. $6^{\text {th }}$ ed. Vol 1. Chap 30. pp 740-748

4. Cole BR,Salinas-Madrigal L. Acute proliferative glomerulonephritis and crescentic glomerulonephritis. In: Holliday MA, Baratt TM, Avner ED,eds. Pediatric Nephrology, $3^{\text {rd }}$ edn. Williams and Wilkins;1994, 694-718. 
5. Khoybar MA, Sultana S, Shaha AK, Anwar MH, Ashraful MI, Ruhul AS, Jahangir HB. Immediate outcome of acute glomerulonephritis in children experience in a tertiary level hospital. Bangladesh Journal of Medical Science. 2011 Oct. Vol. 10 No. 04.

6. Glassock RJ, Cohen Ah, Adler SG. Primary Glomerular Diseases. Brenner and Reetor's. The Kidney, 5th ed. Saunders, 2:1392-1473

7. Sarkissian A et al. An epidemic of Acute Postinfectious GN in Armenia. Arch. Dis. Child 1997 Oct; 77(4):342-4.

8. Streeton CL, Hanna JN, Messer RD, Merianos A. An epidemic of APSGN among aboriginal children. JPaediatr-child-Health 1995; 31(3):245-8.

9. Richards J. Acute post-streptococcal glomerulonephritis. W V Med J 1991; 87:61-5.

10. Lewy JE, Salinas-Madrigal L, Herdson PB, Pirani $\mathrm{CL}$,Metcoff J. Clinicopathologic correlations in acute poststreptococcal glomerulonephritis. Medicine (Baltimore) 1971; 50:453-501

11. Vilmar de Paiva Marques, Precil Diego Miranda, de Menezes Neves, Helena Moises Mendonca,
Itsuzi Fugikaha, Edson Luiz Fernandes. Acute glomerulonephritis after upper airway or skin infection: descriptive analysis of 82 cases between 14and 64 years-old. J Bras Nefrol 2010; 32(3):237240

12. Fabiola D Cruz et al. Acute glomerulonephrtis in Kelantan- a prospective study. Med. J. Malaysia 1990 June Vol. 45 No.2

13. Chugh KS et al. Progression to end stage renal disease in post-streptococcal glomerulonephritis (PSGN)---Chandigarh Study. Int J Artif Organs. 1987 May; 10(3):189-94.

14. Sarala Rajajee. Post-streptococcal acute glomerulonephritis: A clinical, bacteriological and serological study. The Indian Journal of Pediatrics. 1990 November-December, Volume 57, Issue 6, pp 775-780

15. Shakur MS, Khorshed MS, Dash PK. Skin Lesions, A Major Association Acute Nephritis in Children. DS (Child) H J 2000; 16(2):5-11.

16. Fang GX. Clinical features and long-term outcome of 91 cases of adult onset post-streptococcal glomerulonephritis in Hong Kong. Zhonghua Nei Ke Za Zhi. 1989; 28:486-9. 